\title{
Functional Characterization of Generalized Langevin Equations
}

\author{
Adrián A. Budini ${ }^{1}$ and Manuel O. Cáceres $^{2}$ \\ ${ }^{1}$ Max Planck Institute for the Physics of Complex Systems, \\ Nöthnitzer Str. 38, \\ 01187 Dresden, Germany \\ ${ }^{2}$ Centro Atómico Bariloche, Instituto Balseiro, CNEA, Univ. \\ Nac. de Cuyo and CONICET, \\ Av. E. Bustillo Km 9.5, 8400 Bariloche, Argentina
}

(September 17, 2018)

\begin{abstract}
We present an exact functional formalism to deal with linear Langevin equations with arbitrary memory kernels and driven by any noise structure characterized through its characteristic functional. No others hypothesis are assumed over the noise, neither the fluctuation dissipation theorem. We found that the characteristic functional of the linear process can be expressed in terms of noise's functional and the Green function of the deterministic (memory-like) dissipative dynamics. This object allow us to get a procedure to calculate all the Kolmogorov hierarchy of the non-Markov process. As examples we have characterized through the 1-time probability a noiseinduced interplay between the dissipative dynamics and the structure of different noises. Conditions that lead to non-Gaussian statistics and distributions with long tails are analyzed. The introduction of arbitrary fluctuations in fractional Langevin equations have also been pointed out.
\end{abstract}

Typeset using REVTEX 


\section{INTRODUCTION}

Noise is a basic ingredient of many types of model in physics, mathematics, economy, as well as in engineering. Besides that in each area of research the fluctuations have very different origins, in many cases the evolution equation governing the system of interest can be approximated by a suitable stochastic differential equation.

In general the driving forces may be any source of fluctuations and the system can be characterized by a given potential. The most popular of those stochastic differential equations are the one driven by white Gaussian fluctuations, therefore the problem can immediately be reduced to the well known Fokker-Planck dynamics ${ }^{1-3}$. If the fluctuations are not Gaussian we are faced with a problem that is hard to solve, but among the different types of fluctuations the so call dichotomic noise is a good candidate to study, because in general for any potential some conclusions can be drawn ${ }^{4-8}$. If the fluctuations are different from the Gaussian ones or the dichotomic noise, in general it is not possible to solve the problem for any potential.

In many situations the technical complications of the model can be diminished by studying the system in a linear approximation, i.e., around the fixed points of the dissipative dynamics. This approximation leads to the study of linear stochastic differential equations with arbitrary noises. Besides the simplicity of this kind of equations they were the subject of numerous theoretical investigation ${ }^{9-25}$, and also they provide non-trivial models for the study of many different mechanism of relaxation in physics, biology and another research areas.

From the previous considerations, it is clear that the usefulness of a linear Langevin equation arises from the possibility of working with different kind of noise structures. Therefore, one is faced with the characterization, in general, of non-Markov processes. These pro-

cesses can only be completely characterized through the whole Kolmogorov hierarchy of the stochastic process ${ }^{2}$, i.e.: the knowledge of any $n$-joint probability, or equivalently any $n$-time moment and/or cumulant. 
In Ref. ${ }^{26,27}$, by using a functional technique, we have been able to characterize arbitrary linear Langevin equations with local dissipation, giving therefore a procedure to calculate the whole Kolmogorov hierarchy of the process and any $n$-time moment. Using this previous experience, in this paper we are going to generalize our functional technique to tackle the more general situation where the dissipative term is non-local in time and the noise is also arbitrary; this is what we call a Generalized Langevin Equation, i.e., a linear Langevin equation with arbitrary memory and driven by any noise structure

$$
\frac{d}{d t} u(t)=-\int_{0}^{t} d t^{\prime} \Phi\left(t-t^{\prime}\right) u\left(t^{\prime}\right)+\xi(t), \quad u \in(-\infty,+\infty),
$$

where $\xi(t)$, the fluctuation term (i.e., the external noise) is characterized by its associated functional, and $\Phi(t)$ is an arbitrary memory kernel.

This type of generalized Langevin equation arises quite natural (considering Gaussian fluctuations) in the context of the Zwanzig-Mori projector operator technique ${ }^{28,29}$; in this case the fluctuation-dissipation theorem is required ${ }^{30,31}$, which imposes that $\Phi(t)=$ $\langle\xi(t+\tau) \xi(\tau)\rangle / k T$. Therefore the dissipative memory must be consistent with the structure of the correlation of the Gaussian fluctuations ${ }^{30}$. Nevertheless, if the system is far away from equilibrium, the fluctuation-dissipation theorem is not fulfilled and in general the Gaussian assumption is not a good candidate to describe the fluctuations of the system. Therefore, in a general situation, both the kernel and the noise properties must be considered independent elements whose interplay will determine the full stochastic dynamics of the process $u(t)$. Along the paper we will be interested in characterizing this noise-induced interplay by assuming different kind of noises and memory kernels, both in the transient as in the long-time regime.

The paper is organized as follows. In section II, after a short review of the functional method, we obtain the characteristic functional for a vectorial Langevin equation with memory. In section III we apply this result to different situations. First we analyze the case of stable noises; after this step, we analyze the stochastic dynamics induced by an exponential memory kernel in a process driven by two different fluctuating structures: radioactive 
and Poisson noises. Then the interplay between the kernel and the noise properties is emphasized. The necessary noise's properties that guarantee a stationary distribution with a long-tail are presented. As an example we introduce the Abel noise that induces this asymptotic behavior. The characterization of a fractional Langevin equation with arbitrary noise is also presented. The interplay between the fractional property of the differential operator and the Lévy noise is analyzed. In section IV we give the conclusions.

\section{FUNCTIONAL CHARACTERIZATION OF ARBITRARY LINEAR LANGEVIN EQUATIONS}

\section{A. The characteristic functional method}

In our previous papers, we have presented a complete characterization of Langevin equations with local dissipation by means of the characteristic functional of the stochastic process $u(t)[t \in(0, \infty)]$

$$
G_{u}([k(t)])=\left\langle\exp i \int_{0}^{\infty} d t k(t) u(t)\right\rangle
$$

Here $k(t)$ is a test function, and $\langle\cdots\rangle$ means an average over the stochastic realizations of $u(t)$ belonging to a given support. The knowledge of the characteristic functional allows a full characterization of the process. In fact, from this functional it is possible to calculate the whole Kolmogorov hierarchy, and of course any $n$ - time moment and/or cumulant. This follows by defining the $n$-characteristic function $G_{u}^{(n)}\left(\left\{k_{j}, t_{j}\right\}_{j=1}^{n}\right)$ as

$$
G_{u}^{(n)}\left(\left\{k_{j}, t_{j}\right\}_{j=1}^{n}\right)=G_{u}\left(\left[k_{\delta}(t)\right]\right)
$$

where the test function $k_{\delta}(t)$ must be taken as

$$
k_{\delta}(t)=k_{1} \delta\left(t-t_{1}\right)+\cdots+k_{n} \delta\left(t-t_{n}\right)
$$

Thus, any $n$-time joint probability distribution $P\left(\left\{u_{j}, t_{j}\right\}_{j=1}^{n}\right) \equiv P_{n}\left(u_{1}, t_{1} ; u_{2}, t_{2} \cdots ; u_{n}, t_{n}\right)$

can be obtained by Fourier inversion of $G_{u}^{(n)}\left(\left\{k_{j}, t_{j}\right\}_{j=1}^{n}\right)$ 


$$
P\left(\left\{u_{j}, t_{j}\right\}_{j=1}^{n}\right)=\frac{1}{(2 \pi)^{n}} \int d k_{1} \cdots \int d k_{n} \exp \left(-i \sum_{j=1}^{n} k_{j} u_{j}\right) G_{u}^{(n)}\left(\left\{k_{j}, t_{j}\right\}_{j=1}^{n}\right) .
$$

On the other hand, any $n$-time moment can be calculated as

$$
\left\langle u\left(t_{1}\right) u\left(t_{2}\right) \cdots u\left(t_{n}\right)\right\rangle=\left.(-i)^{n} \frac{\partial^{n} G_{u}^{(n)}\left(\left\{u_{j}, t_{j}\right\}_{j=1}^{n}\right)}{\partial k_{1} \partial k_{2} \cdots \partial k_{n}}\right|_{k_{j}=0} .
$$

An equivalent formulae holds for the cumulants (or correlation functions), in which case the differentiation must be taken from the logarithm of the $n$-characteristic function

$$
\left\langle\left\langle u\left(t_{1}\right) u\left(t_{2}\right) \cdots u\left(t_{n}\right)\right\rangle\right\rangle=\left.(-i)^{n} \frac{\partial^{n} \ln G_{u}^{(n)}\left(\left\{u_{j}, t_{j}\right\}_{j=1}^{n}\right)}{\partial k_{1} \partial k_{2} \cdots \partial k_{n}}\right|_{k_{j}=0} .
$$

We have showed ${ }^{26,27}$ that in order to obtain the characteristic functional of $u(t)$, it is only necessary to know the characteristic functional of the noise $\xi(t)$

$$
G_{\xi}([k(t)])=\left\langle\exp i \int_{0}^{\infty} d t k(t) \xi(t)\right\rangle
$$

No any other hypothesis were assumed over the noise. This general formalism allows us to deal with arbitrary non-Markovian evolution processes, where there is not a clear underlying Fokker-Planck dynamics.

In what follows we will generalize our functional characterization for the case of a Langevin dynamics that includes memory effects, Eq. (1.1). We remark that not any condition as thermal equilibrium, Gaussian noises, or any other, is imposed on the stochastic evolution of the processes $u(t)$.

\section{B. The multivariable linear case with dissipative memory}

A general case that covers many models of evolution, is the vectorial linear stochastic equation

$$
\frac{d}{d t} \mathbf{u}(t)=-\int_{0}^{t} d t^{\prime} \mathbf{\Phi}\left(t-t^{\prime}\right) \cdot \mathbf{u}\left(t^{\prime}\right)+\xi(t)
$$

Here, $\mathbf{u}(t)$ represent a $d$-dimensional stochastic process $\left\{u_{i}(t)\right\}_{i=1}^{d}$, with $u_{i} \in(-\infty,+\infty)$. The memory kernel $\boldsymbol{\Phi}(t)$ is an $d \times d$ matrix with arbitrary kernel functions. The $d$-dimensional vector noise $\xi(t)$ is characterized through its characteristic functional 


$$
G_{\xi}([\mathbf{k}(t)])=\left\langle\exp i \int_{0}^{\infty} d t \mathbf{k}(t) \cdot \xi(t)\right\rangle
$$

where the $\operatorname{dot}\{\cdot\}$ denotes a scalar vectorial product, and $\mathbf{k}(t)$ is a vector of test functions $\left\{k_{i}(t)\right\}_{i=1}^{d}$.

We want to found an exact expression for the functional of the process $\mathbf{u}(t)$, defined as

$$
G_{\mathbf{u}}([\mathbf{k}(t)])=\left\langle\exp i \int_{0}^{\infty} d t \mathbf{k}(t) \cdot \mathbf{u}(t)\right\rangle
$$

The basic idea consists in writing the solution of Eq. (2.8) in terms of the corresponding Green function. By denoting the Laplace transform as $\tilde{f}(s)=\int_{0}^{\infty} e^{-s t} f(t) d t$, from Eq. (2.8) we get

$$
s \tilde{\mathbf{u}}(s)-\mathbf{u}(0)=-\tilde{\boldsymbol{\Phi}}(s) \cdot \tilde{\mathbf{u}}(s)+\tilde{\xi}(s) .
$$

This allows to express the formal solution for each realization of the noises as

$$
\mathbf{u}(t)=\langle\mathbf{u}(t)\rangle_{0}+\int_{0}^{t} d t^{\prime} \boldsymbol{\Lambda}\left(t-t^{\prime}\right) \cdot \xi\left(t^{\prime}\right)
$$

where we have defined the vector

$$
\langle\mathbf{u}(t)\rangle_{0} \equiv \boldsymbol{\Lambda}(t) \cdot \mathbf{u}(0)
$$

The $d \times d$ matrix $\boldsymbol{\Lambda}(t)$ is defined through its Laplace transform

$$
\tilde{\boldsymbol{\Lambda}}(s)=\frac{1}{s \hat{\mathbf{I}}+\tilde{\boldsymbol{\Phi}}(s)}
$$

where $\hat{\mathbf{I}}$ is a $d$-dimensional identity matrix and $\tilde{\boldsymbol{\Phi}}(s)$ is the Laplace transform of the matrix kernel $\boldsymbol{\Phi}(t)$. Eq. (2.14) is equivalent to the evolution

$$
\frac{d}{d t} \boldsymbol{\Lambda}(t)=-\int_{0}^{t} d t^{\prime} \boldsymbol{\Lambda}\left(t^{\prime}\right) \cdot \boldsymbol{\Phi}\left(t-t^{\prime}\right), \quad \boldsymbol{\Lambda}(0)=1,
$$

From this equation it is possible to identify the matrix $\boldsymbol{\Lambda}(t)$ with the Green function of Eq. (2.8). Notice that the vector $\langle\mathbf{u}(t)\rangle_{0}$ corresponds to the average value of the process $\mathbf{u}(t)$ for the case in which the average of the noise is null, i.e., $\langle\xi(t)\rangle=0$. 
After introducing the solution Eq. (2.12) into Eq. (2.10), and reordering the time integrals $\int_{0}^{t} d t^{\prime} \int_{0}^{t^{\prime}} d t^{\prime \prime}=\int_{0}^{t} d t^{\prime \prime} \int_{0}^{t} d t^{\prime} \Theta\left(t^{\prime}-t^{\prime \prime}\right)$, where $\Theta(t)$ is the step function, we arrive to a closed expression for the characteristic functional of $\mathbf{u}(t)$ in terms of the functional of the vector noise. Thus, we get

$$
G_{\mathbf{u}}([\mathbf{k}(t)])=G_{\langle\mathbf{u}\rangle_{0}}([\mathbf{k}(t)]) G_{\xi}([\mathbf{z}(t)]),
$$

where we have defined

$$
G_{\langle\mathbf{u}\rangle_{0}}([\mathbf{k}(t)])=\exp \left\{i \int_{0}^{\infty} d t \mathbf{k}(t) \cdot\langle\mathbf{u}(t)\rangle_{0}\right\}
$$

and the vector test function $\mathbf{z}(t)$ as

$$
\mathbf{z}(t)=\int_{t}^{\infty} d t^{\prime} \mathbf{k}\left(t^{\prime}\right) \cdot \mathbf{\Lambda}\left(t^{\prime}-t\right)
$$

The expression Eq. (2.16) gives us the desired exact functional $G_{\mathbf{u}}([\mathbf{k}(t)])$ as the product of two functional. The first one corresponds to the deterministic evolution, or equivalently to the averaged process when the noises have null averages. On the other hand, the second term comes from the noise characteristic functional, i.e., the stochastic evolution.

Finally, the $n$-time characteristic function $G_{\mathbf{u}}^{(n)}\left(\left\{\mathbf{k}_{j}, t_{j}\right\}_{j=1}^{n}\right)$ can immediately be evaluated from the characteristic functional of the noise. The unidimensional case, Eq. (2.2), is easily generalized to the vectorial case as

$$
G_{\mathbf{u}}^{(n)}\left(\left\{\mathbf{k}_{j}, t_{j}\right\}_{j=1}^{n}\right)=G_{\mathbf{u}}\left(\left[\mathbf{k}_{\delta}(t)\right]\right),
$$

where the vectorial test function $\mathbf{k}_{\delta}(t)$ is

$$
\mathbf{k}_{\delta}(t)=\mathbf{k}_{1} \delta\left(t-t_{1}\right)+\cdots+\mathbf{k}_{n} \delta\left(t-t_{n}\right)
$$

Thus, using these last two equations and Eq. (2.16) to (2.18), we get

$$
G_{\mathbf{u}}^{(n)}\left(\left\{\mathbf{k}_{j}, t_{j}\right\}_{j=1}^{n}\right)=\exp \left\{i \sum_{j=1}^{n} \mathbf{k}_{j} \cdot\left\langle\mathbf{u}\left(t_{j}\right)\right\rangle_{0}\right\} G_{\xi}([\mathbf{y}(t)]),
$$

where the function $\mathbf{y}(t)$ reads 


$$
\mathbf{y}(t)=\sum_{j=1}^{n} \Theta\left(t_{j}-t\right) \mathbf{k}_{j} \cdot \boldsymbol{\Lambda}\left(t_{j}-t\right)
$$

Eq. (2.21) gives, in a simple way, the $n$-characteristic function of the process $\mathbf{u}(t)$ by evaluating the characteristic functional of the noise with the function $\mathbf{y}(t)$. This last function is defined in terms of the Green function $\boldsymbol{\Lambda}(t)$ of the problem. At this point, it is important to remark that our formalism is valid independently of the assumed form for the dissipative Green function evolution. Thus, this function may present a monotonous or oscillating decay (taking positive and negative values), and in general any one consistent with the corresponding kernel. In Appendix A we show a particular 2-dimensional stochastic process $\left\{u_{i}(t)\right\}_{i=1}^{2}$ where each component $u_{i}(t)$ represents the position and the velocity of a memory damped harmonic oscillator in presence of an arbitrary noise structure.

\section{EXAMPLES}

Here we are going to analyze, and characterize with our functional method, many amazing situations that arise by choosing different noise structures and a non-local dissipative kernel.

\section{A. Local Dissipation}

From now on, we will restrict to a unidimensional case. By assuming a $\delta$-Dirac correlated kernel

$$
\Phi(t)=\gamma \delta(t)
$$

we arrive to the evolution

$$
\frac{d}{d t} u(t)=-\gamma u(t)+\xi(t)
$$

Therefore, it is possible to obtain $\tilde{\Lambda}(s)=1 /(s+\gamma)$. Thus,

$$
\Lambda(t)=\exp [-\gamma t]
$$

With this solution, it is possible to recapture all the results obtained in Ref. $^{26}$. 


\section{B. Stable noises}

Stable probability distributions play an important role in the theory of sums of random variables. This fact follows from the generalized central limit theorem valid for Lévy distributions and the asymptotic power-law decay ${ }^{32,33}$. Here we are going to apply our functional formalism with the use of stable noises.

\section{Gaussian noise}

A zero-mean Gaussian noise $\xi(\tau)$, with an arbitrary correlation function $\sigma_{\xi}\left(\tau_{2}, \tau_{1}\right)=$ $\left\langle\xi\left(\tau_{2}\right) \xi\left(\tau_{1}\right)\right\rangle$, is characterized by the functional ${ }^{2}$

$$
G_{\xi}([k(t)])=\exp \left(-\frac{1}{2} \int_{0}^{\infty} d \tau_{2} \int_{0}^{\infty} d \tau_{1} k\left(\tau_{2}\right) \sigma_{\xi}\left(\tau_{2}, \tau_{1}\right) k\left(\tau_{1}\right)\right) .
$$

Therefore, from Eqs. (2.16) to (2.18) the characteristic functional of the process $u(t)$ results

$$
G_{u}([k(t)])=G_{\langle u\rangle_{0}}([k(t)]) \exp \left(-\frac{1}{2} \int_{0}^{\infty} d \tau_{2} \int_{0}^{\infty} d \tau_{1} k\left(\tau_{2}\right) k\left(\tau_{1}\right) \sigma_{u}\left(\tau_{2}, \tau_{1}\right)\right)
$$

where

$$
\sigma_{u}\left(\tau_{2}, \tau_{1}\right)=\int_{0}^{\tau_{2}} d \tau_{b} \int_{0}^{\tau_{1}} d \tau_{a} \Lambda\left(\tau_{2}-\tau_{b}\right) \sigma_{\xi}\left(\tau_{b}, \tau_{a}\right) \Lambda\left(\tau_{1}-\tau_{a}\right)
$$

is the correlation function of the Gaussian process $u(t)$. In fact, the $n$-time characteristic function of $u(t)$ is a multivariate Gaussian process.

\section{Lévy noise}

Another example that share similar conclusions than using the Gaussian noise, is the Lévy noise. The characteristic functional of this noise reads ${ }^{21}$

$$
G_{\xi}([k(t)])=\exp \left(-\frac{\sigma_{\nu}}{2} \int_{0}^{\infty} d \tau|k(\tau)|^{\nu}\right)
$$

where $0<\nu<2$. Thus, for example, the 1 -time characteristic function of the process $u(t)$ reads 


$$
G_{u}^{(1)}(k, t)=\exp \left\{i k\langle u(t)\rangle_{0}\right\} \exp \left(-\frac{1}{2}|k|^{\nu} \Xi_{u}(t)\right)
$$

where

$$
\Xi_{u}(t)=\sigma_{\nu} \int_{0}^{t} d t^{\prime}\left|\Lambda\left(t^{\prime}\right)\right|^{\nu}
$$

Therefore, memory-like linear Langevin equations driven by stable noises give rise to new stable stochastic processes whose correlations properties are defined in terms of the Green function $\Lambda(t)$ of the dissipative dynamics. Note that this result is valid independently of the particular form of the Green function.

\section{Interplay between non-local friction and noise structure}

In general, around a fixed point, the linearized dynamics of a dissipative systemdepending on the parameters of the problem - may presents a relaxation behavior with quite different characteristics: let us say, a monotonous or a time-oscillating decay. Here we are going to study the relaxation and the fluctuations of the process $u(t)$ by analyzing the interplay between these mentioned dissipative behaviors and different noise structures.

The different behaviors of the dissipative dynamics can be modeled through a non-local exponential memory friction

$$
\Phi(t)=\delta \exp [-\lambda t]
$$

therefore $\tilde{\Phi}(s)=\delta /(s+\lambda)$. From Eq. (2.14) we get $\tilde{\Lambda}(s)=(s+\lambda) /[s(s+\lambda)+\delta]$, which after inversion gives

$$
\Lambda(t)=e^{-\lambda t / 2}\left\{\cos \left[w_{0} t\right]+\frac{\lambda}{2 w_{0}} \sin \left[w_{0} t\right]\right\}
$$

where the frequency $w_{0}$ is

$$
w_{0}=\sqrt{\delta-\left(\frac{\lambda}{2}\right)^{2}}
$$


As desired, the dissipative Green function, Eq.(3.11), has a regime where it decays oscillating in time. But on the other hand there is a possibility, for $\delta<\left(\frac{\lambda}{2}\right)^{2}$ where $\Lambda(t)$ decays in a monotonous way. Note that the local case (non-memory dissipation), Eq. (3.1), is reobtained considering the limit: $\lambda \rightarrow \infty$ and $\delta \rightarrow \infty$, with $\delta / \lambda \rightarrow \gamma$ (finite).

Now we are going to present some results using the Green function Eq. (3.11) in connection with two different structures for the fluctuating term, i.e., a radioactive noise and a Poisson white-noise. We will show that the interplay between the deterministic Green function and the structure of the noise play a crucial role to determine the relaxation and in general any statistical dynamical property of the stochastic process $u(t)$. On the other hand, a memory kernel with a power-law decay will be studied in the context of fractional derivatives in the sub-section E.

\section{Radioactive decay noise}

The radioactive noise is non-white and Markovian. Its 1-time and conditional probability evolve controlled by the master equation

$$
\frac{d P_{\xi}(t)}{d t}=\beta\left[(\xi+1) P_{\xi+1}(t)-\xi P_{\xi}(t)\right]
$$

Thus, the realizations of this noise start with some initial value $\xi_{0}$ and at random times decrease by finite unit steps until the process reaches the zero value. The constant $\beta$ defines the probability per unit of time for such discrete steps $(\beta$-decay). The characteristic functional reads ${ }^{2}$

$$
G_{\xi}([k(t)])=\left(\beta \int_{0}^{\infty} d \tau \exp \left[-\beta \tau+i \int_{0}^{\tau} d \tau^{\prime} k\left(\tau^{\prime}\right)\right]\right)^{\xi_{0}}
$$

This noise clearly does not reach a stationary regime. Using this noise, the $1-$ time characteristic function of the process $u(t)$ results

$$
G_{u}^{(1)}(k, t)=e^{i k\langle u(t)\rangle_{0}}\left(e^{-\beta t} \exp \left[i k \int_{0}^{t} d \tau^{\prime} \Lambda\left(\tau^{\prime}\right)\right]+\beta \int_{0}^{t} d \tau e^{-\beta \tau} \exp \left[i k \int_{t-\tau}^{t} d \tau^{\prime} \Lambda\left(\tau^{\prime}\right)\right]\right)^{\xi_{0}}
$$


This noise leads to very striking dynamical behaviors for the driven process $u(t)$. In the following figures we have plotted, at different times, the corresponding exact probability distribution $P(u, t)$. In order to get this object we have anti-transformed Eq. (3.15) by using a fast Fourier algorithm. In Fig. 1, the parameters for the memory kernel were chosen as $\delta=0.1, \lambda=1$. Thus, the Green function decays without oscillations (see inset). The noise decay rate is $\beta=1$ and its initial value was chosen to be $\xi_{0}=2$. At time $t=1$ (full line), we see that the probability distribution is highly irregular and also has a $\delta$-Dirac contribution (indicated symbolically with the vertical line). This singular behavior arises from the particular realization $\xi(t)$ in which the noise has not yet suffer any decay.

Due to the non-vanishing value of the noise average, $\langle\xi(t)\rangle \neq 0$, the $\delta$-Dirac contribution has different locations at different times. This term can be directly read from the characteristic functional of the process $u(t)$, Eq. (3.15), which gives: $\exp \left(-\xi_{0} \beta t\right) \delta\left[u-\xi_{0} \int_{0}^{t} d \tau^{\prime} \Lambda\left(\tau^{\prime}\right)\right]$. The discontinuity of the probability distribution $P(u, t)$ is due to the strong change that arises in the driving noise after a $\beta$-decay. In fact, in this example, the noise intensity decreases to half of its initial value. At a later time, $t=5$ (dotted line), due to the dissipative dynamics the probability distribution $P(u, t)$ loss it discontinuous character and seems to be accumulated near of the origin. At even later time, $t=15$ (dashed line) this accumulation seems to increase. As a matter of fact, the stationary distribution is a $\delta$-Dirac centered in $u=0$. This behavior is a consequence of the coupling between the noise properties and the deterministic dynamics of $u(t)$ : as the noise intensity dead at long times, all realizations $u(t)$ are attracted by the stable point of the dissipative dynamics, which corresponds to $u=0$.

In Fig. 2 we show the case in which the Green function is oscillatory in time (see inset). The parameters are $\delta=0.1, \lambda=0.2$, and for the noise we choose $\beta=1$ and $\xi_{0}=10$. Here, in contrast to the previous figure, we have increased the initial intensity of the noise, which imply that the $\delta$-Dirac contribution decay in a very fast way. On the other hand, this higher initial value imply that the "discrete" decay of the corresponding noise realizations do not change appreciably the noise intensity. In consequence, at all time the distribution $P(u, t)$ results smooth. As in the previous example, all realization of the process $u(t)$ are 
attracted by the stable point $u=0$. Nevertheless, here the transient behavior reflects the oscillatory behavior of the Green function. In fact, we note that at successive times, the center of mass of the distribution $P(u, t)$ approximately follows the dissipative dynamics of the Green function oscillating around the origin $u=0$ (see times: $t=1$ continuous line, $t=5$ dotted line, $t=15$ dashed line, $t=25$ dot-dashed line). In addition, we note that the width of the distribution grows approximately up to $t=5$. In fact, at this time most of the noise realizations have decayed to a null value. For later times, the distribution is mainly governed by the dissipative dynamics of $u(t)$. Therefore, its width decreases up to reaching a $\delta$-Dirac form in the stationary state.

We remark that for this noise the stationary distribution $P(u)$ is always determinate by the fixed point of the dissipative dynamics. This characteristic is due to the vanishing noise's amplitude at the long time regime. In the next example we show a case where the noise properties participate, in a crucial way, both in the transient as in the stationary properties of the process $u(t)$.

\section{Shot noise}

The stochastic realizations of the shot noise are defined by a sequence of pulses $\psi(t)$, each

one arriving at random independent times $t_{i}$. The characteristic functional of the noise $\mathrm{e}^{2,26,27}$ $\xi(t)$, in the interval $t \in[0, \infty]$, is

$$
G_{\xi}([k(t)])=\exp \left(\int_{0}^{\infty} d \tau q(\tau)\left[\exp \left(i \int_{0}^{\infty} k(t) \psi(t-\tau) d t\right)-1\right]\right)
$$

where $q(\tau)$ is the density of arriving pulses. Therefore, the complete characterization of the process $u(t)$ is given by

$$
G_{u}([k(t)])=G_{\langle u\rangle_{0}}([k(t)]) \exp \left(\int_{0}^{\infty} d \tau q(\tau)\left[\exp \left(i \int_{0}^{\infty} k(t) \Psi(t, \tau) d t\right)-1\right]\right)
$$

where the function $\Psi(t, \tau)$ is defined as

$$
\Psi(t, \tau)=\int_{0}^{t} d t^{\prime} \Lambda\left(t-t^{\prime}\right) \psi\left(t^{\prime}-\tau\right)
$$


If $\psi(t)=A \delta(t)$ and the density of "dots" $q(\tau)$ is uniform $q(\tau)=\rho$ the noise $\xi(t)$ reduces to the more familiar white shot-noise or Poisson noise whose characteristic functional is

$$
G_{\xi}([k(t)])=\exp \left(\rho \int_{0}^{\infty} d t[\exp [i A k(t)]-1]\right) .
$$

By adding two statistically independent white shot-noises, with opposite amplitudes, we get

$$
G_{\xi}([k(t)])=\exp \left(2 \rho \int_{0}^{\infty} d t[\cos (A k(t))-1]\right) .
$$

This functional characterizes a noise with a null average, whose realizations consists in the random arriving of $\delta$-Dirac pulses with amplitude $\pm A$. Note that in the limit $A \rightarrow 0$, $\rho \rightarrow \infty$ with $A^{2} \rho=D / 2$, this symmetrical white shot-noise converges to a Gaussian white noise, i.e., Eq. (3.4) with $\sigma_{\xi}\left(\tau_{2}, \tau_{1}\right)=D \delta\left(\tau_{2}-\tau_{1}\right)$.

Using Eq. (3.20), the 1-time characteristic function of the process $u(t)$ is given by

$$
G_{u}^{(1)}(k, t)=\exp \left\{i k\langle u(t)\rangle_{0}\right\} \exp \left(2 \rho \int_{0}^{t} d \tau[\cos (A k \Lambda(\tau))-1]\right) .
$$

As we will show below, this expression leads to a rich variety of possible stochastic dynamical behavior for the process $u(t)$; both, in the transient as in the stationary state, the different behaviors arise from the competence between the different characteristic time scales of the Green function [Eq. (3.11)] and those of the noise. In the monotonous regime, $\delta<\lambda^{2} / 4$, the decay of $\Lambda(\tau)$ can be characterized by the rate $\delta / \lambda$. For $\delta>\lambda^{2} / 4$, when $w_{0}>\lambda / 2$, the oscillatory behavior of $\Lambda(\tau)$ is characterized by the damping rate $\lambda / 2$ and the frequency $w_{0}$. On the other hand, the noise is characterized the rate $\rho$ and the amplitude $A$. The analysis of the behavior of $P(u, t)$ on these parameters can be simplified by defining the rescaled process $u^{\prime}(t)=u(t) / A$, whose evolution can be written as

$$
\frac{d u^{\prime}\left(\tau^{\prime}\right)}{d \tau^{\prime}}=-\delta^{\prime} \int_{0}^{\tau^{\prime}} d \tau^{\prime \prime} \exp \left[-\left(\tau^{\prime}-\tau^{\prime \prime}\right)\right] u^{\prime}\left(\tau^{\prime \prime}\right)+\xi^{\prime}\left(\tau^{\prime}\right) .
$$

Here, we have defined the dimensionless time $\tau^{\prime}=\lambda t$ and parameter $\delta^{\prime}=\delta / \lambda^{2}$. Furthermore, the dimensionless symmetric Poisson noise $\xi^{\prime}\left(\tau^{\prime}\right)$ is only characterized by the dimensionless rate $\rho^{\prime}=\rho / \lambda$. Thus, the full dynamical properties of the process $u(t)$ are controlled by the parameters $\delta^{\prime}$ and $\rho^{\prime}$. 
In the next figures we have obtained numerically $P(u, t)$ from fast Fourier transform of Eq. (3.21). In Fig. 3 the parameters of the kernel are $\delta=0.2, \lambda=1$, and for the noise we used $A=1, \rho=7.5$. For these values, the frequency $w_{0}$ in Eq. (3.12) is complex. Therefore, the Green function $\Lambda(t)$ decays monotonously in time (see inset). At short times, $t=0.5$ [Fig. 3(a)], the probability distribution presents a series of peaks, each ones separated by a distance $A=1$. This property is a direct consequence of the nature of the shot-noise, where each arriving $\delta$-Dirac pulse produces a shift of magnitude $A$ in the driven process $u(t)$. In this short time regime the effect of the dissipative dynamics is negligible. At later times, the dissipative contribution becomes appreciable; its effects is to attract all the realization of $u(t)$ to the fixed point $u=0$. This action, added to the shift effect of the arriving pulses, produces an increasing of the width of all the peaks of the distribution; then any value of $u$ becomes (approximately) probable. This effect is clearly seen in Fig. 3(b) $[t=1.65]$. Note that the distribution retained the "sharpy" structure of peaks. This erasing effect is even increased with time; see Fig. 3(c) (time $t=2)$. At later times, all the signature of the peaks have disappeared and the distribution goes asymptotically to a Gaussian distribution; see Fig. 3(d) [times $t=2.5$ (dotted line) and $t=20$ (full line)].

In general, an asymptotic Gaussian stationary distribution is always expected when the rate of the arriving $\delta$-Dirac pulses is greater than the characteristic decay rate of the Green function. When the decay of $\Lambda(t)$ is monotonous, this condition can be expressed through the inequality $\rho^{\prime}>\delta^{\prime}$, which is clearly satisfied in the previous figure. When this relation is not satisfies, deviations from a Gaussian distribution must arise both in the transient as in the stationary distribution. We show this effect in the next figures.

In Fig. 4, the parameters of the Green function (see inset) are the same than in the previous figure, $[\delta=0.2, \lambda=1]$, and for the noise we have used $A=1, \rho=0.1$. As in the previous case, the behavior of $P(u, t)$ arises from the competence between the shift effect of the arriving pulses and the dissipative dynamics of $u(t)$. For example, at short times [Fig. 4(a), $t=2.5]$ the probability shows a set of maxima located at $\pm A$ with tails pointing in the direction to the origin. These tails are mainly originated by the dissipative 
dynamics that attracts all realizations to the stable point $u=0$. On the other hand, here the occupation around $u=0$ comes from the shift effect produced by two arriving pulses with different signs. In contrast to the previous example, at later times [Fig. 4(b), $t=5$ ] the evolution of the distribution $P(u, t)$ is mainly governed by the dissipative dynamics and the structure of peaks is retained during all the evolution, Fig. 4(c) $t=10$, and Fig. 4(d) $t=100$, where the stationary state is practically reached. Note that in this stationary state, the non-Gaussian characteristics are present in $u \approx 0$ and in $u \approx \pm A$.

We note that during all the transient dynamics, there exist a $\delta$-Dirac contribution (indicated with the vertical lines) centered at the origin, $u=0$. This term is originated by the noise realization in which not any pulse has arrived up to time $t$. Therefore, it is exponentially damped with a rate $2 \rho$, i.e., it can be written as $\exp (-2 \rho t) \delta(u)$. At each of the chosen times, the weight of the $\delta$-Dirac term are respectively: $0.606,0.368,0.135$ and $2 \times 10^{-9}$. Notice that in the stationary state, the $\delta$-Dirac contribution is always completely washed out. We remark that, independently of the values of the parameters, this $\delta$-Dirac contribution is always present. Nevertheless, note that in the case of Fig. 3, it is rapidly attenuated and its contribution is insignificant in the time scale of that plot.

In the two previous figures we have assumed a monotonous decreasing Green function. In general the interplay between an asymmetric Poisson noise, Eq. (3.19), and an oscillatory Green function is similar to that founded in the case of the Radioactive noise. In the case of a symmetric noise, Eq. (3.21), due to the symmetry of the problem, the transient dynamics is approximately similar to that founded for a monotonous decay. Thus, the more relevant aspect to analyze is the corresponding stationary distribution.

In Fig. 5 we study the properties of the stationary distribution $P(u)$ for different values of the parameter of the Green function and the noise. In Fig. 5(a) the parameters corresponding to the Green function (see inset) are $\delta=2, \lambda=1$, while for the Poisson noise we have taken $\rho=0.3$ (dotted line), $\rho=0.4$ (dashed line) and $\rho=0.8$ (continuous line); in all cases taking $A=\sqrt{0.4 / \rho}$. In consequence, the effective amplitude of the noise $\rho A^{2}$ is constant in the three graphs. Due to this selection the tails of the distributions coincide, indicating that all 
the distributions are asymptotically, for large $|u|$, Gaussian. This property will be analyzed analytically in the next-subsection.

As in the case of the non-oscillating Green function, Figs. 3 and 4, here we expect a Gaussian stationary distribution when the average waiting time between two arriving pulses is shorter than the characteristic relaxation time of the Green function. In this case, the oscillatory decay of the Green function is characterized by the rate $\lambda / 2$. Thus, we estimate that for $\rho^{\prime}>1 / 2$ a Gaussian statistics arises. This prediction agree very well with the plots shown in Fig. 5(a). Nevertheless, note that this inequality does not depend on the frequency of the Green function oscillations. In the next figure, we will check the validity of this prediction.

In Fig. 5(b) we show the stationary distribution $P(u)$ by maintaining the noise parameters and changing the values of the parameters of the Green function. The noise parameters were chosen as $\rho=0.25, A=1$ and for the Green function $\lambda=1, \delta=0.15$ (full line), $\delta=0.6$ (dashed line), and $\delta=50$ (dotted line). In the inset we show the corresponding Green function for each values. Consistently with our previous analysis, we confirm that independently of the value of the characteristic frequency of the Green function, the stationary distribution $P(u)$ is non Gaussian for $\rho^{\prime}<1 / 2$. Furthermore, we note that by increasing the frequency of the Green function, the fast oscillations of $\Lambda(t)$ completely drop-out the non-Gaussian peaks located at $\pm A$, remaining only one peak centered at $u=0$. On the other hand, with the chosen values of the noise parameters, a Gaussian distribution only arise for a non-oscillating Green function.

Finally, we want to remark that the criteria for obtaining a stationary Gaussian distribution $\rho^{\prime}>\delta^{\prime}$, valid when the decay of the Green function is monotonous, and $\rho^{\prime}>1 / 2$ valid for an oscillatory decay, change their validity in a smooth way around the value $\delta^{\prime}=1 / 4$, which corresponds to the point in which the Green function modify its characteristic decay. 


\section{Long-tail stationary distributions}

Here we are interested in characterizing the stationary distribution of the stochastic process $u(t)$; of great importance is to know whether there will be or not a long-tail in the distribution. In order to make a general analysis, here we assume that the structure of the noise is such that we can write the 1 -time characteristic function in the form

$$
G_{u}^{(1)}\left(k_{1}, t_{1}\right)=\exp \left\{\int_{0}^{t_{1}} d t f\left(k_{1} \Lambda(t)\right)\right\}
$$

where $f(z)$ is an arbitrary function. Note that this structure is compatible only with a white noise. In fact, Eq. (3.23) follows using the test function $z(t)=\Theta\left(t_{1}-t\right) \Lambda\left(t_{1}-t\right) k_{1}$ in the general expression for the functional of $u(t)$, Eq. (2.16), and assuming $G_{\xi}[k(t)]=$

$\exp \int_{0}^{\infty} d t f(k(t))$. Taking the logarithm in Eq. (3.23), and introducing the Laplace transform (here denoted as $\mathcal{L}_{s}$ ) we arrive to the general expression

$$
\ln G_{u}^{(1)}(k, t=\infty)=\lim _{s \rightarrow 0} s \mathcal{L}_{s}\left[\ln G_{u}^{(1)}(k, t)\right]=\lim _{s \rightarrow 0} \mathcal{L}_{s}[f(k \Lambda(t))]
$$

If a Taylor expansion of $f(z)$ exists, and we can commute the Taylor expansion and the Laplace operator, we arrive to

$$
\ln G_{u}^{s t}(k)=\sum_{n=1}^{\infty} C_{n} k^{n} \mathcal{L}_{s}\left[\Lambda(t)^{n}\right]_{s=0} .
$$

This formulae characterizes the stationary distribution of the stochastic process $u(t)$, if it exists, as a series expansion in the Fourier component $k$.

From Eq. (3.25) it is simple to see that even when the stochastic transient can be nonGaussian (depending on the driving noise) in the large asymptotic scaling $|u| \rightarrow \infty$, the Fourier transform of the stationary distribution goes like

$$
G_{u}^{s t}(k) \sim \exp \left(i k A_{0}-k^{2} B_{0}+\cdots\right), \text { for } k \sim 0
$$

where $A_{0}, B_{0}$ are constants given in terms of the dissipative memory and the structure of the noises; then at large scale the behavior is not anomalous. The analysis with a non-white 
noise is similar and the general conclusion is not changed. So in order to understand the occurrence of anomalies or long-tails in the stationary distribution of the process $u(t)$ we have to consider driving noises that break the hypothesis Eq. (3.25). This case is achieved by structures like the one from the Lévy noise, see Eq. (3.7), or its associated one-side power-law noise shown in appendix B.

In the previous sub-section we have shown that the Poisson noise, Eq. (3.19), can give rise, during the transient and in the stationary state, to strongly non-Gaussian distributions for the process $u(t)$. Nevertheless, this noise can not give rise to long-tail stationary distributions. As a matter of fact, the characterization of the stationary state induced by this shot noise follows straightforward using Eq. (3.24). From Eq. (3.19) its follows

$$
\begin{aligned}
\ln G_{u}^{s t}(k) & =\lim _{t \rightarrow \infty} \rho \int_{0}^{t} d \tau(\exp [i A k \Lambda(\tau)]-1), \\
& =\frac{\rho}{\gamma}\{\operatorname{Ei}(i A k)-\ln (A|k|)-\mathcal{E}\},
\end{aligned}
$$

where $\operatorname{Ei}(x)$ is the exponential integral function ${ }^{34}$, and $\mathcal{E}$ is the Euler constant. For simplifying the analysis we have assumed the local Green function Eq. (3.3). Now it is interesting to remark about the non-analytic structure of $G_{u}^{s t}(k)$, perhaps pre-announcing an anomalous behavior in the stationary distribution of the process $u(t)$. Nevertheless, it should be noted that in the large scale limit $k \rightarrow 0$ this non-analytic structure cancel out, leading therefore to an expansion like Eq. (3.26), which imply the absence of any long-tail distributions. This is not what happens if the driving noise has a long-tail structure as the one we are going to show below.

\section{Abel noise}

In close connection with a Poisson noise with a random density of arriving pulses, in appendix B we have defined the characteristic functional of Abel noise $\xi(t)$. The functional of this noise reads $[t \in(0, \infty)]$

$$
G_{\xi}([k(t)])=\frac{2}{\Gamma(\mu)}\left(\sqrt{a \int_{0}^{\infty}\left(1-e^{i k(t)}\right) d t}\right)^{\mu} K_{\mu}\left(2 \sqrt{a \int_{0}^{\infty}\left(1-e^{i k(t)}\right) d t}\right)
$$


where $K_{\mu}(z)$ is the Basset function and $\Gamma(\mu)$ the Gamma function ${ }^{34}$. Note that in contrast with the Lévy noise, this functional has well defined integers moments $\left\langle\xi(t)^{q}\right\rangle$ if $\mu>q$. Using this noise, the 1 -time characteristic function of the process $u(t)$ reads

$$
G_{u}^{(1)}(k, t)=\frac{2 e^{i k\langle u(t)\rangle_{0}}}{\Gamma(\mu)}\left(\sqrt{a \int_{0}^{t}\left(1-e^{i k \Lambda(\tau)}\right) d \tau}\right)^{\mu} K_{\mu}\left(2 \sqrt{a \int_{0}^{t}\left(1-e^{i k \Lambda(\tau)}\right) d \tau}\right) .
$$

From this expression (if $\Lambda(t)>0, \forall t$ ) it is possible to see that the asymptotic behavior of the probability distribution is characterized by a one-side power-law distribution of the Abel form

$$
P(u, t) \sim \frac{\mathcal{A}(t)^{\mu}}{\Gamma(\mu)} \frac{\exp (-\mathcal{A}(t) / u)}{u^{1+\mu}}, \text { for large positive } u
$$

where

$$
\mathcal{A}(t)=a \int_{0}^{t} \Lambda(\tau) d \tau>0
$$

In the case of local dissipation, using the Green function Eq. (3.3), we get

$$
\mathcal{A}(t)=\frac{a}{\gamma}\left(1-e^{-\gamma t}\right)
$$

From this result it is simple to see that the (one-side stable) directed random walk behaves like $P(u, t) \sim(a t)^{\mu} / u^{1+\mu}$, as was expected; see appendix B.

In presence of a non-local dissipation and in the case $\delta>\left(\frac{\lambda}{2}\right)^{2}$, due to the fact that the Green function Eq.(3.11) changes its sign oscillating in time, a power-law behavior is obtained in a quite unusual way during the transient, i.e., the long-tail changes its support from a positive to a negative domain and so on as the time goes on. On the other hand, in the stationary regime we get

$$
\mathcal{A}(t=\infty)=\frac{a \lambda}{\delta}
$$

then also a power-law is reached at long times, with its long-tail in the positive domain, i.e., for $u>0, P(u, t \rightarrow \infty) \sim\left(\frac{a \lambda}{\delta}\right)^{\mu} u^{-1-\mu}$. 
In Fig. 6 we show this amazing behavior for the noise parameters $a=3, \mu=\frac{1}{2}$, $K_{1 / 2}(x)=\sqrt{\pi / 2 x} \exp (-x)$. In this case we have used for the Green function (see inset) the parameters values $\delta=0.85$ and $\lambda=0.2$. At time $t=3.5$ [Fig. 6(a)] we can see that there is a long-tail in the positive domain, but also a sort of exponential decay appears in the negative domain as a result of the interplay between the oscillating Green function and the Abel noise. At later time $t=5[$ Fig. 6(b)] it is possible to see that the long-tail has changed its support, showing also an exponential decay but now in the positive domain. At time $t=50$ [Fig. 6(c)] the stationary distribution is almost reached with the expected long-tail in the positive domain. In these figures we have also fitted (with dashed lines) the corresponding long-tails with the asymptotic behavior

$$
P(u, t) \sim\left|\frac{\Gamma(-\mu)}{\Gamma(\mu)}\right| \frac{|\mathcal{A}(t)|^{\mu}}{[\operatorname{sig}(u) \cdot u]^{1+\mu}},
$$

where $\operatorname{sig}(u)$ represents the sign of $u$.

To end this sub-section let us call the attention that by subtracting two statistically independent Abel noises, it is also possible to obtain the $n$-time characteristic function of a symmetric process $u(t)$, see appendix B.

\section{E. Fractional derivative evolutions}

Another example that can be characterized with the present functional technique is the case of fractional Langevin equations with dissipation. There exist many different way of introducing this kind of equations. Here we will consider the evolution

$$
{ }_{0} D_{t}^{\alpha}[u(t)]-u_{0} \frac{t^{-\alpha}}{\Gamma(1-\alpha)}=-\eta^{\alpha} u(t)+\xi(t)
$$

with $1 \geq \alpha>0$. This evolution was proposed to simulate the dynamics of financial markets

where it was found that non-Gaussian driving noises should be the suitable ones ${ }^{23,24}$. The proper interpretation of this fractional stochastic equation is actually an integral equation

$$
u(t)-u_{0}=-\eta^{\alpha}{ }_{0} D_{t}^{-\alpha}[u(t)]+{ }_{0} D_{t}^{-\alpha}[\xi(t)] .
$$


Here, $u_{0}$ is the initial condition, and ${ }_{0} D_{t}^{-\alpha}$ is the Riemann-Liouville integral operator ${ }^{35}$

$$
{ }_{0} D_{t}^{-\alpha}[f(t)]=\frac{1}{\Gamma(\alpha)} \int_{0}^{t} d \tau \frac{f(\tau)}{(t-\tau)^{1-\alpha}} .
$$

It has been proved ${ }^{23}$ that the solution of this equation for each realization of the noises can be written as

$$
u(t)=\langle u(t)\rangle_{0}+\int_{0}^{t} d t^{\prime} \Lambda\left(t-t^{\prime}\right) \xi\left(t^{\prime}\right)
$$

where

$$
\langle u(t)\rangle_{0}=u_{0} E_{\alpha, 1}\left[-(\eta t)^{\alpha}\right]
$$

On the other hand, the Green function $\Lambda(t)$ is given by

$$
\Lambda(t)=\Theta(t) t^{\alpha-1} E_{\alpha, \alpha}\left[-(\eta t)^{\alpha}\right]
$$

Note that for this model of dissipative fractional equations the average $\langle u(t)\rangle_{0}$ and the noise propagate with different Green functions. The generalized Mittag-Leffler function $E_{\alpha, \beta}$ is defined by

$$
E_{\alpha, \beta}(z)=\sum_{k=0}^{\infty} \frac{z^{k}}{\Gamma(\alpha k+\beta)} \quad \alpha>0, \beta>0 .
$$

From Eq. (3.37) it is evident that our functional approach allows us to introduce any kind of statistics to drive the fractional stochastic evolution.

\section{Competition between fractional derivative and Lévy noise}

Here we are interested in analyzing the competence between the statistic of Lévy noise and the dynamical effects introduced by a fractional derivative structure. In order to get a simpler analysis, here we will assume zero dissipation, i.e., $\eta=0$. In this case, the previous evolution reduce to

$$
\langle u(t)\rangle_{0}=u_{0}, \quad \Lambda(t)=\frac{\Theta(t)}{\Gamma(\alpha)} \frac{1}{t^{1-\alpha}}
$$


Note that from differentiation of Eq. (3.35), these two previous expressions characterizes the evolution

$$
\frac{d u(t)}{d t}={ }_{0} D_{t}^{1-\alpha} \xi(t)
$$

We would like to stress that this problem in the frame of our functional approach can be done in a simple way. Using Eq. (3.41) and the functional of a Lévy white noise, Eq. (3.7), the process $u(t)$ is fully characterized by the function [see Eq. (3.9)]

$$
\Xi_{u}(t)=\sigma_{\nu}\left(\frac{1}{\Gamma(\alpha)}\right)^{\nu}\left(\frac{1}{1-\nu(1-\alpha)}\right) t^{1-\nu(1-\alpha)}
$$

This expression is only well defined if $\nu(1-\alpha)<1$. Note the restriction on the parame-

ters $\{\nu, \alpha\}$. This result contrasts with that of a driving Gaussian white noise, where the restriction is $\alpha \in\left(\frac{1}{2}, 1\right]$. Thus, in the case of Lévy noise, smaller values of the parameter $\alpha \in(0,1]$ are allowed at the expenses of diminishing the value of $\nu \in(0,2]$ (i.e., large Lévy-step excursions).

Finally, we want to remark that Eq. (3.42) driven by Gaussian fluctuations, can be mapped with an effective medium approximation in the context of disordered systems ${ }^{3,36}$. Note that from our functional approach, it is easy to calculate any correlation functions and more complicated objects than the propagator of the system; this is something that is hard to obtain in the context of self-consistent approximations ${ }^{37}$.

\section{SUMMARY AND CONCLUSIONS}

We have completely characterized generalized linear Langevin equations when the usual fluctuation-dissipation theorem does not apply. Our central result is an exact expression for the characteristic functional of the process $u(t)$, for a general $d$-dimensional correlated process (in appendix A we present a 2-dimensional example) where the memory and the noise are arbitrary. In this way we have been able to give a closed expression to get the whole Kolmogorov hierarchy, i.e., to calculate any $n$-joint probability and any $n$-time correlation function or cumulant. 
We have applied our formalism to many different noise structures, and in this way we have shown the noise-induced interplay between the effects of the dissipative memory and the structure of the driving noises. Particular emphasis have been put in analyzing the 1-time probability distribution $P(u, t)$ and its stationary state; we have shown that the transient toward the stationary state strongly depends on the interplay between the dissipation and the noise structure. As example we have analyzed the case of an exponential memory function in presence of different stochastic driving forces; for example, radioactive noise and Poisson noise. Also we have discussed whether or not, at large scale, a non-Gaussian distribution appears and when these distributions have long-tails. In order to get this class of stationary distributions we have introduced, for the first time, the Abel noise in connection with the occurrence of one-side power-law distributions (see appendix B). We remark that this noise structure can be useful when studying models where molecular diffusion is an important ingredient to be considered. In this context, the relaxation analysis of a generalized Langevin particle considering memory dissipation and injection of energy by microscopic random contributions characterized by a symmetric power-law, can be also carried out by using the present functional formalism. As a matter of fact, asymptotically when the Abel noise is symmetric the conclusions, for $\mu<2$, are in agreement with the structure of the Lévy noise.

Of particular relevance is the use of our formalism to study fractional Langevin equations driven by arbitrary noise structures. We have analyzed the competence between the noise statistic and the fractional derivative operator. As examples we have compared Lévy and Gaussian noises and their interplay with the fractional calculus. We remark that the possibility of introducing arbitrary noise statistics in fractional Langevin equations is an interesting step forward to broad the possible applications of these equations ${ }^{23}$.

To end this work let us comment that the present functional approach can also be applied to the so called delayed Langevin equations. In that case the associated Green function $\Lambda(t)$ turns to be the crucial ingredient to study different models of delayed Langevin equations driven by arbitrary noises. Results along this line will be presented elsewhere. 


\section{APPENDIX A: A TWO DIMENSIONAL EXAMPLE}

In what follows we will show a multivariable example that can be fully worked out by using our formalism. It corresponds to a particle confined in an harmonic potential and driven by an arbitrary noise $\xi(t)$ (fluctuating force). The evolution for the position $x(t)$ and the velocity $v(t)$ reads

$$
\begin{aligned}
& \frac{d}{d t} x(t)=v(t) \\
& \frac{d}{d t} v(t)=-\Omega^{2} x(t)-\int_{0}^{t} d t^{\prime} \Phi\left(t-t^{\prime}\right) v\left(t^{\prime}\right)+\xi(t),
\end{aligned}
$$

where $\Omega$ is the characteristic frequency of the harmonic potential. All statistical information of the processes $[x(t), v(t)]$ is encoded in the characteristic functional

$$
G_{x v}\left(\left[k_{x}(t), k_{v}(t)\right]\right)=\left\langle\exp i \int_{0}^{\infty} d t\left(k_{x}(t) x(t)+k_{v}(t) v(t)\right)\right\rangle .
$$

For each realization of the noise, the solution of Eqs. (A1) and (A2) reads

$$
\begin{aligned}
& x(t)=\langle x(t)\rangle_{0}+\int_{0}^{t} d t^{\prime} \mathfrak{X}\left(t-t^{\prime}\right) \xi\left(t^{\prime}\right), \\
& v(t)=\langle v(t)\rangle_{0}+\int_{0}^{t} d t^{\prime} \mathfrak{F}\left(t-t^{\prime}\right) \xi\left(t^{\prime}\right) .
\end{aligned}
$$

Here, we have defined the averages

$$
\begin{aligned}
\langle x(t)\rangle_{0} & =x(0)+\int_{0}^{t} d t^{\prime}\left\langle v\left(t^{\prime}\right)\right\rangle_{0} \\
\langle v(t)\rangle_{0} & =v(0) \mathfrak{F}(t)-\Omega^{2} x(0) \mathfrak{X}(t) .
\end{aligned}
$$

The functions $\mathfrak{F}(t)$ and $\mathfrak{X}(t)$ are defined from their Laplace transforms

$$
\mathfrak{X}(s)=\frac{\mathfrak{F}(s)}{s}, \mathfrak{F}(s)=\frac{s}{s[s+\Phi(s)]+\Omega^{2}},
$$

which is equivalent to

$$
\begin{aligned}
\frac{d}{d t} \mathfrak{X}(t) & =\mathfrak{F}(t) \\
\frac{d}{d t} \mathfrak{F}(t) & =-\Omega^{2} \mathfrak{X}(t)-\int_{0}^{t} d t^{\prime} \Phi\left(t-t^{\prime}\right) \mathfrak{F}\left(t^{\prime}\right),
\end{aligned}
$$


with the initial conditions $\mathfrak{X}(0)=0$ and $\mathfrak{F}(0)=1$. After inserting Eqs. (A4)-(A5) in the definition Eq. (A3) we arrive to the exact expression

$$
G_{x v}\left(\left[k_{x}(t), k_{v}(t)\right]\right)=G_{\langle x\rangle_{0}}\left(\left[k_{x}(t)\right]\right) G_{\langle v\rangle_{0}}\left(\left[k_{v}(t)\right]\right) G_{\xi}([z(t)]),
$$

where we have defined

$$
\begin{aligned}
& G_{\langle x\rangle_{0}}\left(\left[k_{x}(t)\right]\right)=\exp \left\{i \int_{0}^{\infty} d t k_{x}(t)\langle x(t)\rangle_{0}\right\} \\
& G_{\langle v\rangle_{0}}\left(\left[k_{v}(t)\right]\right)=\exp \left\{i \int_{0}^{\infty} d t k_{v}(t)\langle v(t)\rangle_{0}\right\} .
\end{aligned}
$$

Furthermore, the scalar function $z(t)$ is defined by

$$
z(t)=\int_{t}^{\infty} d t^{\prime} \mathfrak{X}\left(t^{\prime}-t\right) k_{x}\left(t^{\prime}\right)+\int_{t}^{\infty} d t^{\prime} \mathfrak{F}\left(t^{\prime}-t\right) k_{v}\left(t^{\prime}\right) .
$$

Thus, knowing the characteristic functional of the noise allows to obtain the full characteristic functional of the bidimensional process $[x(t), v(t)]$.

Free Brownian motion: By taking $\Omega=0$, the previous case reduces in a trivial way to the case of a free Brownian particle. In this case the evolution of the particle position is given by

$$
\frac{d^{2} x(t)}{d t^{2}}=-\int_{0}^{t} d t^{\prime} \Phi\left(t-t^{\prime}\right) \frac{d x\left(t^{\prime}\right)}{d t^{\prime}}+\xi(t) .
$$

This second order differential equation also arises when modeling a rigid rotator ${ }^{26,6}$. The marginal statistic description of this equation can be obtained from Eq. (A11) after taking $k_{v}(t)=0$. Quantities like $\langle\cos [x(t)]\rangle$ or $\langle\sin [x(t)]\rangle$ follow immediately from the real and imaginary part of the characteristic functional, and more complex stochastic objects can also be calculated analytically. In general, any order differential stochastic equation can be analyzed with our functional formalism after introducing additional stochastic process corresponding to the different derivatives of the original process.

\section{APPENDIX B: ABEL NOISE}

Abel was probably the first to give an application of fractional calculus ${ }^{38}$. He used derivatives of arbitrary order to solve the isochrone problem in classical mechanics, and the 
integral equation he worked out was precisely the one Riemann used to define fractional derivatives. In a modern context, the Abel-type of integral equation can be written in the form

$$
\begin{aligned}
\xi^{2 \mu} P(\xi) & =a^{\mu} \frac{1}{\Gamma(\mu)} \int_{0}^{\xi}(\xi-y)^{\mu-1} P(y) d y, \quad \mu>0, \\
& =a^{\mu}{ }_{0} D_{\xi}^{-\mu} P(\xi)
\end{aligned}
$$

where $a$ is an arbitrary constant. It is interesting to note that the particular class of normalized one-side Lévy-type of probabilities

$$
P(\xi)=\frac{a^{\mu}}{\Gamma(\mu)} \xi^{-1-\mu} \exp (-a / \xi), \text { with } a>0, \xi>0,
$$

are solution of the fractional differential equation Eq. (B1). The characteristic function of the probability density Eq. (B2) can be calculated taking the Laplace transform, then

$$
\begin{aligned}
G_{\xi}(k) & =\langle\exp (i k \xi)\rangle=\mathcal{L}_{s}[P(\xi)]_{s=-i k} \\
& =\frac{2}{\Gamma(\mu)}(\sqrt{-i k a})^{\mu} K_{\mu}(2 \sqrt{-i k a}),
\end{aligned}
$$

where $K_{\mu}(z)$ is the Basset function and $\Gamma(\mu)$ is the Gamma function. Note that if $\mu \in(0,1)$ the asymptotic behavior of the Basset function shows the expected divergencies for integers moments $\left\langle\xi^{q}\right\rangle$, but in general depending on the value of $\mu$ we can get finite moments

$$
\left\langle\xi^{q}\right\rangle=a^{q} \frac{\Gamma(\mu-q)}{\Gamma(\mu)}, \quad \text { for } \quad \mu>q .
$$

We define the characteristic functional of a stochastic process $\xi(t)$, closely related to the power-law distribution Eq. (B2), in the form

$$
G_{\xi}([k(t)])=\frac{2}{\Gamma(\mu)}\left(\sqrt{a \int_{0}^{\infty}\left(1-e^{i k(t)}\right) d t}\right)^{\mu} K_{\mu}\left(2 \sqrt{a \int_{0}^{\infty}\left(1-e^{i k(t)}\right) d t}\right) .
$$

From this expression all the moments of the noise can be calculated. For example the first moments read

$$
\begin{aligned}
\langle\xi(t)\rangle & =a \frac{\Gamma(\mu-1)}{\Gamma(\mu)}, \text { if } \mu>1, \\
\left\langle\xi\left(t_{1}\right) \xi\left(t_{2}\right)\right\rangle & =a \frac{\Gamma(\mu-1)}{\Gamma(\mu)} \delta\left(t_{1}-t_{2}\right)+a^{2} \frac{\Gamma(\mu-2)}{\Gamma(\mu)}, \text { if } \mu>2 .
\end{aligned}
$$


Note that there is a constant term in the correlation function $\left\langle\left\langle\xi\left(t_{1}\right) \xi\left(t_{2}\right)\right\rangle\right\rangle \equiv\left\langle\xi\left(t_{1}\right) \xi\left(t_{2}\right)\right\rangle-$ $\left\langle\xi\left(t_{1}\right)\right\rangle\left\langle\xi\left(t_{2}\right)\right\rangle$,

$$
\left\langle\left\langle\xi\left(t_{1}\right) \xi\left(t_{2}\right)\right\rangle\right\rangle=\frac{a}{(\mu-1)} \delta\left(t_{1}-t_{2}\right)+\left(\frac{a}{(\mu-1)}\right)^{2} \frac{1}{(\mu-2)}, \text { if } \mu>2 .
$$

As expected this constant term decreases for large values of $\mu$. From now on we will call $\xi(t)$ the Abel noise in honor to that brilliant mathematician.

Now in order to have a clear meaning of this noise we introduce an alternative interpretation. Let a stochastic process $n(t)$ be defined by a directed random walk, then for a given value of transition rate $\rho$ we write the master equation

$$
\frac{d P(n, t)}{d t}=\rho[P(n-1, t)-P(n, t)], \quad \rho>0 .
$$

Using the Darling-Siegert theorem ${ }^{39}$ the characteristic functional of the process $n(t)$ can be found by solving the masterly equation

$$
\frac{d \Pi(n, t)}{d t}=i k(t) n \Pi(n, t)+\rho[\Pi(n-1, t)-\Pi(n, t)], \quad \Pi(n, 0)=\delta\left(n-n_{0}\right),
$$

where $k(t)$ is any test function. The characteristic functional of the process $n(t)$ follows from the limit

$$
G_{n}([k(t)])=\lim _{t \rightarrow \infty} \sum_{n=0}^{\infty} \Pi(n, t) .
$$

Using the generating function $\Pi(Z, t)=\sum_{n=0}^{\infty} Z^{n} \Pi(n, t)$, the solution of the generating function of Eq. (B9) is, with the abbreviation $\int_{0}^{t} k\left(t^{\prime}\right) d t^{\prime}=K(t)$,

$$
\Pi(Z, t)=\left(Z e^{i K(t)}\right)^{n_{0}} \exp \left(\int_{0}^{t} \rho\left[Z e^{i K(t)-i K\left(t^{\prime}\right)}-1\right] d t^{\prime}\right),
$$

so using Eq. (B10) we finally arrive to the functional

$$
G_{n}([k(t)])=e^{i K(\infty) n_{0}} \exp \left(\rho \int_{0}^{\infty}\left[e^{i \int_{t}^{\infty} k\left(t^{\prime}\right) d t^{\prime}}-1\right] d t\right)
$$

From this expression it is simple to see that

$$
G_{n}([k(t)])=e^{i K(\infty) n_{0}} G_{\xi}([z(t)])
$$


where $G_{\xi}([z(t)])$ is the functional of the Poisson noise $(A=1)$ evaluated in the test function $z(t)=\int_{t}^{\infty} k\left(t^{\prime}\right) d t^{\prime}$. This means that the Markov process $n(t)$ controlled by Eq. (B8), with a probability rate $\rho$, is equivalent to solve a linear Langevin equation driven by a Poisson noise $\xi(t)$ with a uniform density of arriving pulses $\rho$. Thus we can write the associated stochastic differential equation

$$
\frac{d n}{d t}=\xi(t)
$$

Now, we will assume that the rate $\rho$ in Eq. (B8) is a random variable with a distribution $P(\rho)$. This assumption arises naturally in the context of disordered systems. Therefore, the final functional can be obtained from Eq. (B12) as

$$
\left\langle G_{n}([k(t)])\right\rangle_{P(\rho)}=e^{i K(\infty) n_{0}} \int_{0}^{\infty} G_{\xi}([z(t)]) P(\rho) d \rho .
$$

This integral can be done for many different distributions $P(\rho)$, in particular if we use the Abel distribution Eq. (B2), we get

$$
\int_{0}^{\infty} G_{\xi}([z(t)]) P(\rho) d \rho=\frac{2}{\Gamma(\mu)}\left(\sqrt{a \int_{0}^{\infty}\left(1-e^{i z(t)}\right) d t}\right)^{\mu} K_{\mu}\left(2 \sqrt{a \int_{0}^{\infty}\left(1-e^{i z(t)}\right) d t}\right),
$$

where we have used that $\mathcal{R}_{e}\left[\int_{0}^{\infty}\left(e^{i z(t)}-1\right) d t\right] \leq 0$. This result ends the interpretation of the Abel noise as the noisy term appearing in Eq. (B14), in close connection with the solution of a directed random walk model with a random (power-law distributed) transition rate $\rho$.

Note that the cumulants of the Abel noise are not self-averaging with respect to the cumulants of the Poisson noise and the average over $P(\rho)$. For example using the Poisson functional, see Eq. (3.19) with $A=1$ [or Eq. (B12)], it is simple to show that its second cumulant is $\left\langle\left\langle\xi\left(t_{1}\right) \xi\left(t_{2}\right)\right\rangle\right\rangle=\rho \delta\left(t_{1}-t_{2}\right)$. Then the average of the correlation of the Poisson noise reads

$$
\int_{0}^{\infty} d \rho P(\rho)\left\langle\left\langle\xi\left(t_{1}\right) \xi\left(t_{2}\right)\right\rangle\right\rangle=a \frac{\Gamma(\mu-1)}{\Gamma(\mu)} \delta\left(t_{1}-t_{2}\right)
$$


result that is different from the calculation of the correlation of the Abel noise, see Eq. (B7).

Consider now the subtraction of two Abel noises $\xi^{c, b}(t)$ statistical independent: $\xi(t)=$ $\xi^{c}(t)-\xi^{b}(t)$. In this case, we can write the characteristic functional of the process $\xi(t)$ in the form

$$
\begin{aligned}
G_{\xi}([k(t)]) & =\left\langle\exp \left(i \int_{0}^{\infty} k(t)\left[\xi^{c}(t)-\xi^{b}(t)\right] d t\right)\right\rangle \\
& =G_{\xi^{c}}([k(t)]) G_{\xi^{b}}([-k(t)]),
\end{aligned}
$$

where each functional is given by Eq. (B5). From the properties of the Basset function we can write this formula in a compact form, using the Kelvin functions, to handle this in a more friendly way. Also from this expression, it is simple to see that the first moment of the symmetric Abel noise $\xi(t)$ is null, etc. 


\section{REFERENCES}

${ }^{1}$ S. Chandrasekar in, Selected Papers on Noise and Stochastic Process, N. Wax, Ed. Dover Publ., New York (1954).

${ }^{2}$ N.G. van Kampen, in Stochastic Processes in Physics and Chemistry, Second Edition, North-Holland, Amsterdam, (1992).

${ }^{3}$ M.O. Cáceres, Elementos de estadística de no equilibrio y sus aplicaciones al transporte en medios desordenados, Barcelona, Ed. Reverté S.A. (2003).

${ }^{4}$ W. Horsthemke and R. Lefever, in Noise-induced Transitions, (Springer-Verlag, Berlin, 1984), and reference therein.

${ }^{5}$ B.J. West, K. Linderberg, and V. Seshadri, Phys. 102 A, 470 (1980).

${ }^{6}$ M.O. Cáceres, Phys. Rev. E 67, 016102 (2003).

${ }^{7}$ M. Annunziato, Phys. Rev. E 65, 021113 (2002).

${ }^{8}$ J. Masoliver, Phys. Rev. E 48, 121 (1993).

${ }^{9}$ K.G. Wang, Phys. Rev. A 45, 833 (1992).

${ }^{10}$ J. Heinrichs, Phys. Rev. E 47, 3007 (1993).

11 A. Fulinski, Phys. Rev. E 50, 2668 (1994).

12 A. Drory, Phys. Rev. E 51, 5298 (1995).

13 J. Masoliver and K.G. Wang, Phys. Rev. E 51, 2987 (1995).

14 J.M. Porra, K.G. Wang, and J. Masoliver, Phys. Rev. E 53, 5872 (1996).

15 S.I. Denisov, W. Horsthemke, Phys. Rev. E 62, 7729 (2000).

${ }^{16}$ T. Srokowski, Phys. Rev. Lett. 85, 2232 (2000).

17 T. Srokowski, Phys. Rev. E. 64, 031102 (2001). 
${ }^{18}$ R. Morgado, F. Oliveira, G.G. Batrouni, and A. Hansen, Phys. Rev. Lett. 89, 100601 (2002).

${ }^{19}$ B.J. West, and V. Seshadri, Phys. 113 A, 203 (1981).

${ }^{20}$ S. Jespersen, R. Metzler and H.C. Fogedby, Phys. Rev. E 59, 2736 (1990).

${ }^{21}$ M.O. Cáceres, J. Phys A 32 , 6009 (1999).

22 M.O. Cáceres, Phys. Rev. E 60, 5208 (1999).

${ }^{23}$ S. Picozzi and B.J. West, Phys. Rev. E 66, 046118 (2002).

${ }^{24}$ P. Gopikrishnan, V. Plerou, L.A. Nunes Amaral, M. Meyer, and H.E. Stanley, Phys. Rev. E 60, 5305 (1999).

${ }^{25}$ R. Reigada, A.H. Romero, K. Lindenberg, and J.M. Sancho, J. Chem. Phys. 111, 676 (1999).

${ }^{26}$ M.O. Cáceres and A.A. Budini, J. Phys. A Math. Gen. 30, 8427, (1997).

${ }^{27}$ A.A. Budini and M.O. Cáceres, J. Phys. A Math. Gen. 32, 4005, (1999).

${ }^{28}$ R. Zwanzig, in Lectures in Theoretical Physics, Boulder, Vol. III, Ed. W.E. Broton (Interscience: 1961, N.Y.).

${ }^{29}$ H. Mori, Phys. Rev. 112, 1892 (1958).

${ }^{30}$ R. Kubo, Rep. Prog. Phys. 29, 255 (1966).

${ }^{31}$ M. Toda, R. Kubo and N. Saitô, in Statistical Physics I, Equilibrium Statistical Mechanics, Springer-Verlag Berlin, Heildelberg (1983).

32 P. Lévy, Paris, Gauthier, Villars, 1954.

${ }^{33}$ B.V. Gnedenko and A.N. Kolmogorov, in Limit distributions for sum of independent random variables. Reading, Addison Wesley, 1954. 
34 J. Spanier and K.B. Oldham, in An Atlas of Functions, Springer-Verlag Berlin, Heildelberg (1987).

${ }^{35}$ R. Metzler and J. Klafter, Phys. Rep. 339, 1 (2000).

36 This mapping can be done through the corresponding generalized diffusion coefficient $D_{\alpha}(s)=\left(s^{2} / 2\right) \mathcal{L}_{s}[\langle\langle u(t) u(t)\rangle\rangle]$, which in the present case reads

$$
D_{\alpha}(s)=\left(\frac{\kappa_{\alpha}}{\Gamma(\alpha)}\right)^{2}\left(\frac{\Gamma(2 \alpha)}{2 \alpha-1}\right) \frac{s^{2(1-\alpha)}}{2}, \alpha \in\left(\frac{1}{2}, 1\right] .
$$

On the other hand from an effective medium approximation ${ }^{37}$, the self-consistent Laplacedependent diffusion coefficient in $1 D$ is given, asymptotically, by

$$
D(s) \approx\left(\frac{\sin \pi \zeta}{2 \pi(1-\zeta)}\right)^{2 /(2-\zeta)} s^{\zeta /(2-\zeta)}
$$

Thus, it is simple to see that in the long time limit $(s \sim 0)$ there is map between the fractional index $\alpha$ and the exponent $\zeta$ that characterizes the strength of the disorder

$$
\alpha=\frac{4-3 \zeta}{4-2 \zeta} \Rightarrow \alpha \in\left(\frac{1}{2}, 1\right]
$$

Note that our functional technique also allows us to calculate more complicated objects in disordered systems; for example, we can calculate the non trivial two-particles density $P\left(u_{1}, t_{1} ; u_{2}, t_{2}\right)$, etc.

${ }^{37}$ S. Alexander, J. Bernasconi, W.R. Schneider, R. Orbach, Rev. Mod. Phys. 53, 175, (1981).

${ }^{38}$ N.H. Abel, "Solution de quelques problems á l'aide díntegrales définies", Werke 1, 10, (1823).

${ }^{39}$ N.G. van Kampen, Phys. Lett. 76A, 104 (1980). 


\section{FIGURES}

FIG. 1. Probability distribution, $P(u, t)$, for a memory-like driven process $u(t)$ with an exponential dissipative kernel and in presence of a Radioactive noise, $\xi(t)$, as a function of $u$ for three different times, $t=1$ (full line), $t=5$ (dotted line), $t=15$ (dashed line), taken in arbitrary units. The initial condition was chosen as $u(t=0)=0$. The noise parameters are $\beta=1$ and $\xi_{0}=2$. The parameters of the Green function are $\delta=0.1$ and $\lambda=1$; the inset shows its monotonous decaying behavior as a function of time. The straight line in $P(u, t=1)$ indicates the $\delta$-Dirac contribution (at short times) in the distribution of the process $u(t)$. Asymptotically $P(u, t \rightarrow \infty)$ goes to a $\delta$-Dirac located at $u=0$.

FIG. 2. Probability distribution $P(u, t)$ as in Fig. 1 for four different times, $t=1$ (continuous line), $t=5$ (dotted line), $t=15$ (dashed line), $t=25$ (dot-dashed line), in arbitrary units. The noise parameters are $\beta=1$ and $\xi_{0}=10$. The parameters of the Green function are $\delta=0.1$ and $\lambda=0.2$; the inset shows its oscillatory decaying behavior as a function of time. Here, at large times the distribution $P(u, t \rightarrow \infty)$ also goes asymptotically to a $\delta$-Dirac located at $u=0$.

FIG. 3. Probability distribution, $P(u, t)$, for a memory-like driven process $u(t)$ with an exponential dissipative kernel and in presence of a symmetric white Poisson noise, $\xi(t)$, as a function of $u$ for five different times, Fig. 3(a) $t=0.5$, Fig. 3(b) $t=1.65$, Fig. 3(c) $t=2$, Fig. 3(d) $t=2.5$ (dotted line) and $t=20$ (full line), taken in arbitrary units. The initial condition was chosen as $u(t=0)=0$. The noise parameters are $A=1, \rho=7.5$. The parameters of the Green function are $\delta=0.2, \lambda=1$; the inset in Fig. 3(d) shows its monotonous decaying behavior as a function of time.

FIG. 4. Probability distribution $P(u, t)$ as in Fig. 3, for four different times, Fig. 4(a) $t=2.5$, Fig. 4(b) $t=5$, Fig. 4(c) $t=10$, and Fig. $4(\mathrm{~d}) t=100$ where the stationary state is practically reached. The noise parameters are $A=1$ and $\rho=0.1$. The parameters of the Green function are as in Fig. 3; the inset in Fig. 4(d) shows its monotonous decaying behavior as a function of time. The $\delta$-Dirac contributions (see text) are indicated with straight lines. 
FIG. 5. Stationary probability distribution, $P(u)$, for a memory-like driven process $u(t)$ with an exponential dissipative kernel and in presence of a symmetric white Poisson noise, as a function of $u$ for different values of the parameters for the Green function and the noise. In Fig. 5(a) $\delta=2$, $\lambda=1$, while for the Poisson noise we take $\rho=0.3$ (dotted line), $\rho=0.4$ (dashed line) and $\rho=0.8$ (continuous line) and $A=\sqrt{0.4 / \rho}$. In Fig. 5(b) the noise parameters are the same in each plot, $\rho=0.25, A=1$, but we change the parameters for the Green function, $\lambda=1, \delta=0.15$ (full line), $\delta=0.6$ (dashed line), and $\delta=50$ (dotted line). The insets show the Green functions for the corresponding different parameters.

FIG. 6. Probability distribution, $P(u, t)$, for a memory-like driven process $u(t)$ with an exponential dissipative kernel and in presence of the Abel noise, $\xi(t)$, as a function of $u$ for three different times in arbitrary units, Fig. 6(a) $t=3.5$, Fig. 6(b) $t=5$, Fig. 6(c) $t=50$. The initial condition was $u(t=0)=0$. The noise parameters are $a=3$ and $\mu=\frac{1}{2}$. The parameters of the Green function are $\delta=0.85$ and $\lambda=0.2$; the inset shows its oscillatory decaying behavior as a function of time. The dashed lines correspond to the long-tail fit (see text). In Fig. 6(c) the stationary regime of the distribution of the process $u(t)$ has been reached. 
Figure 1

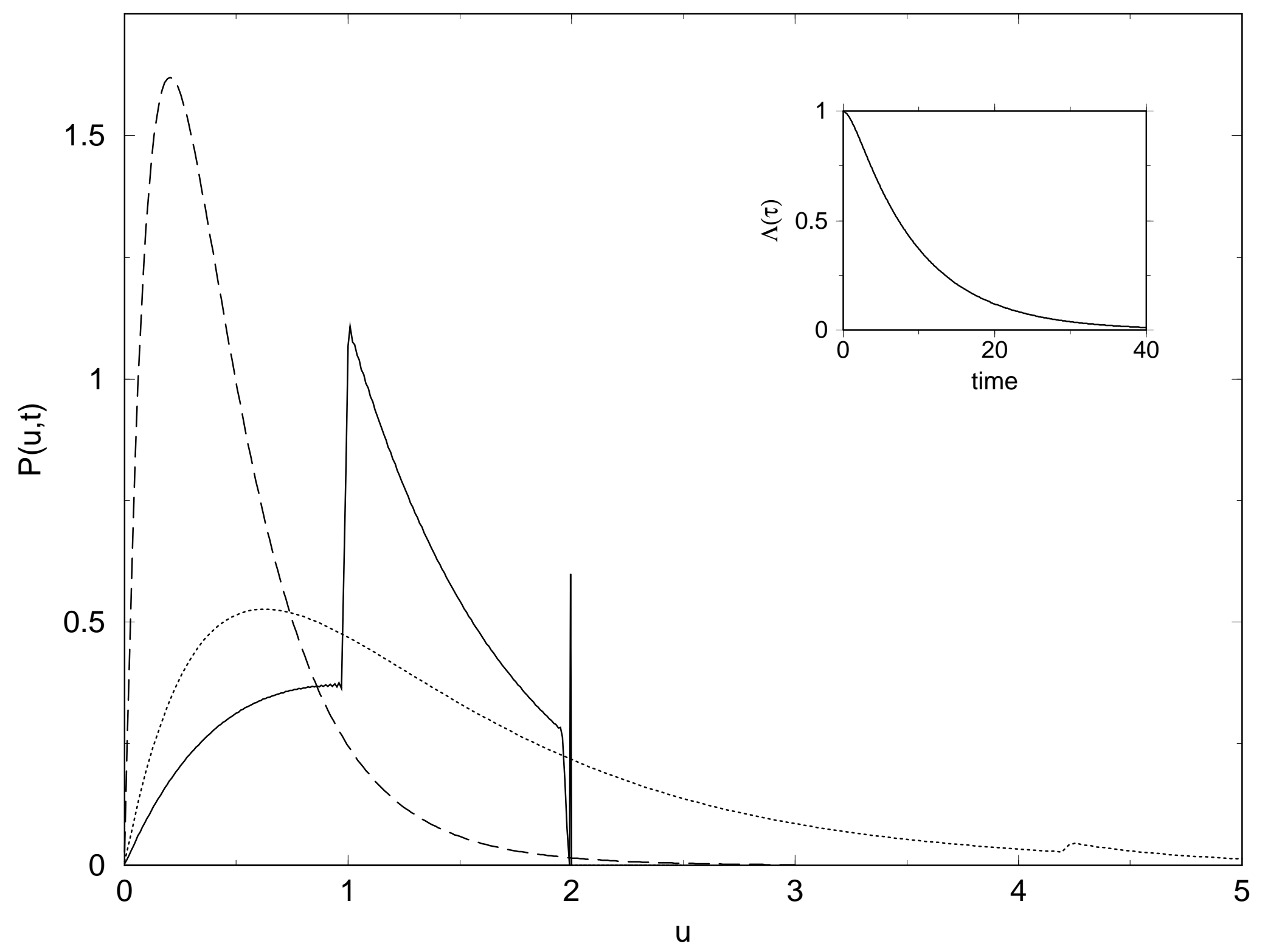


Figure 2

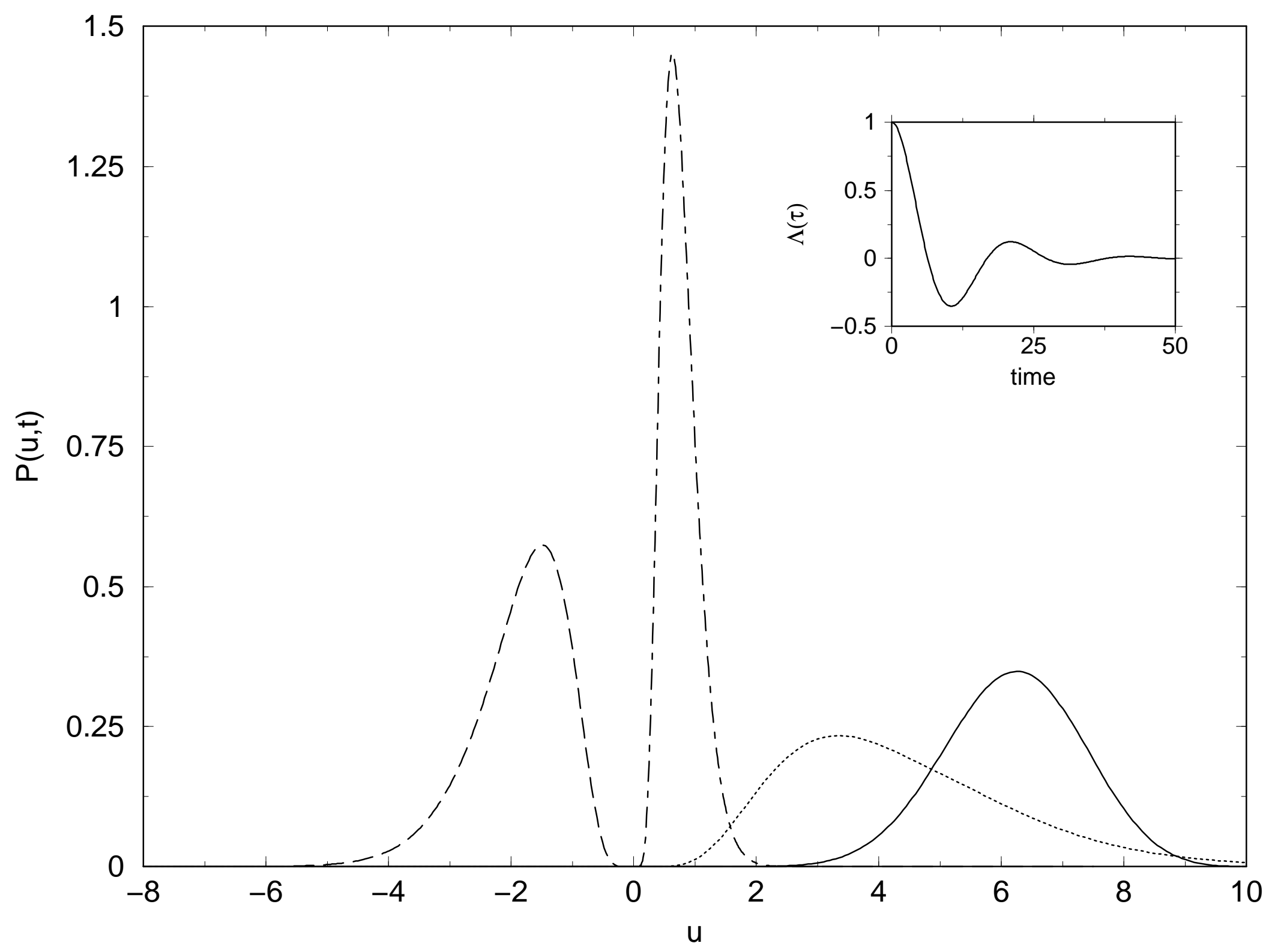


Figure 3
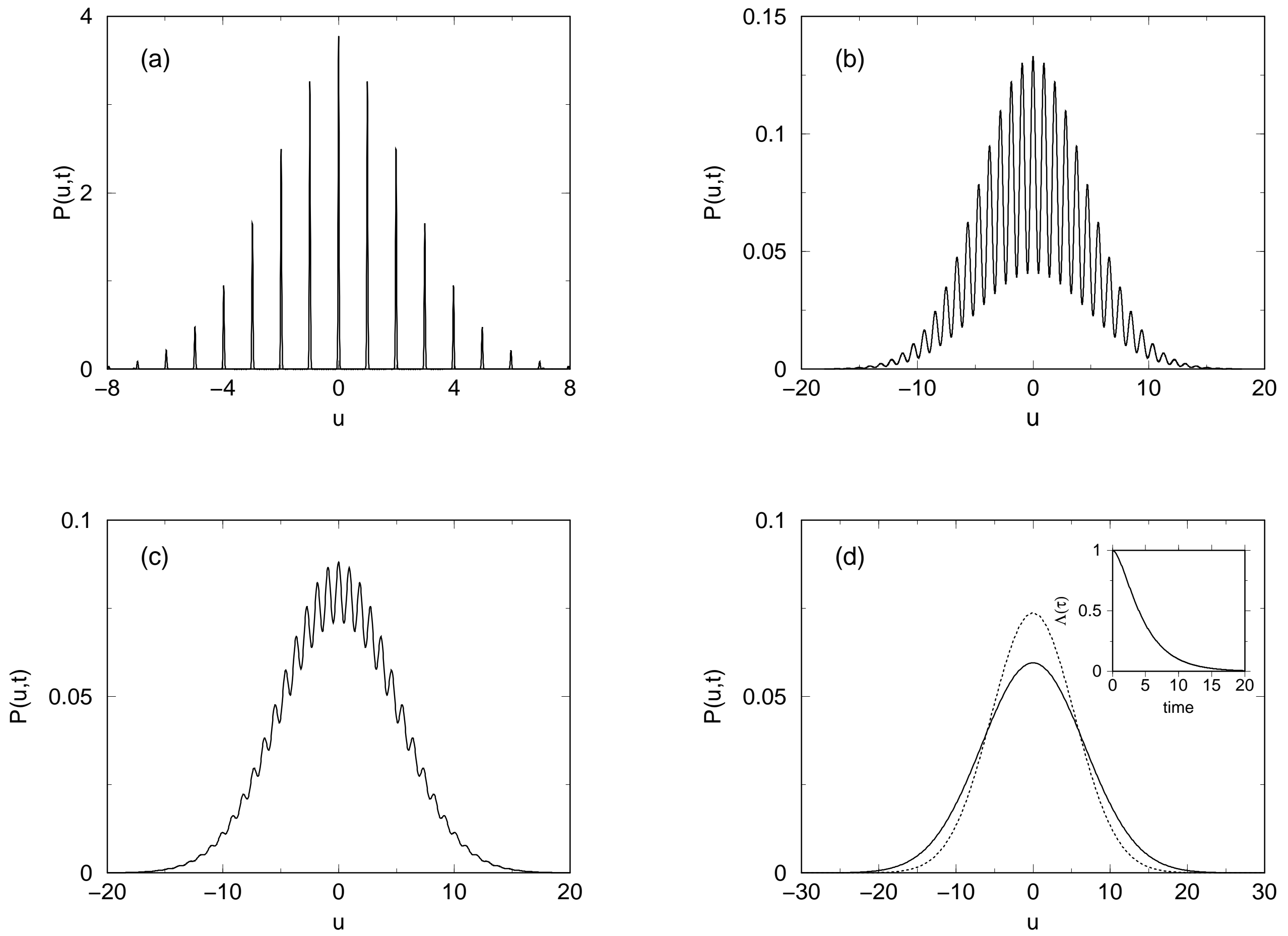
Figure 4
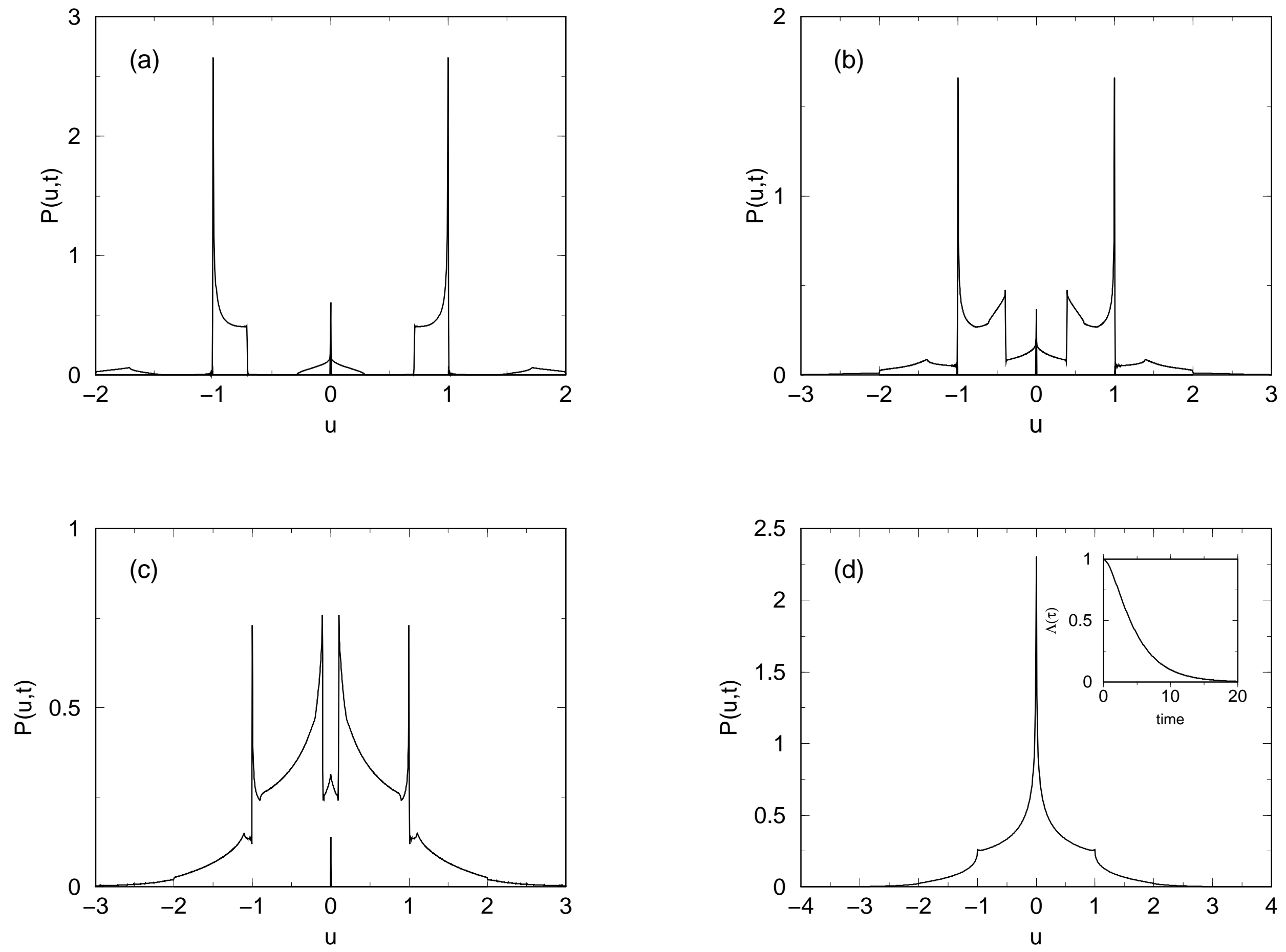
Figure 5
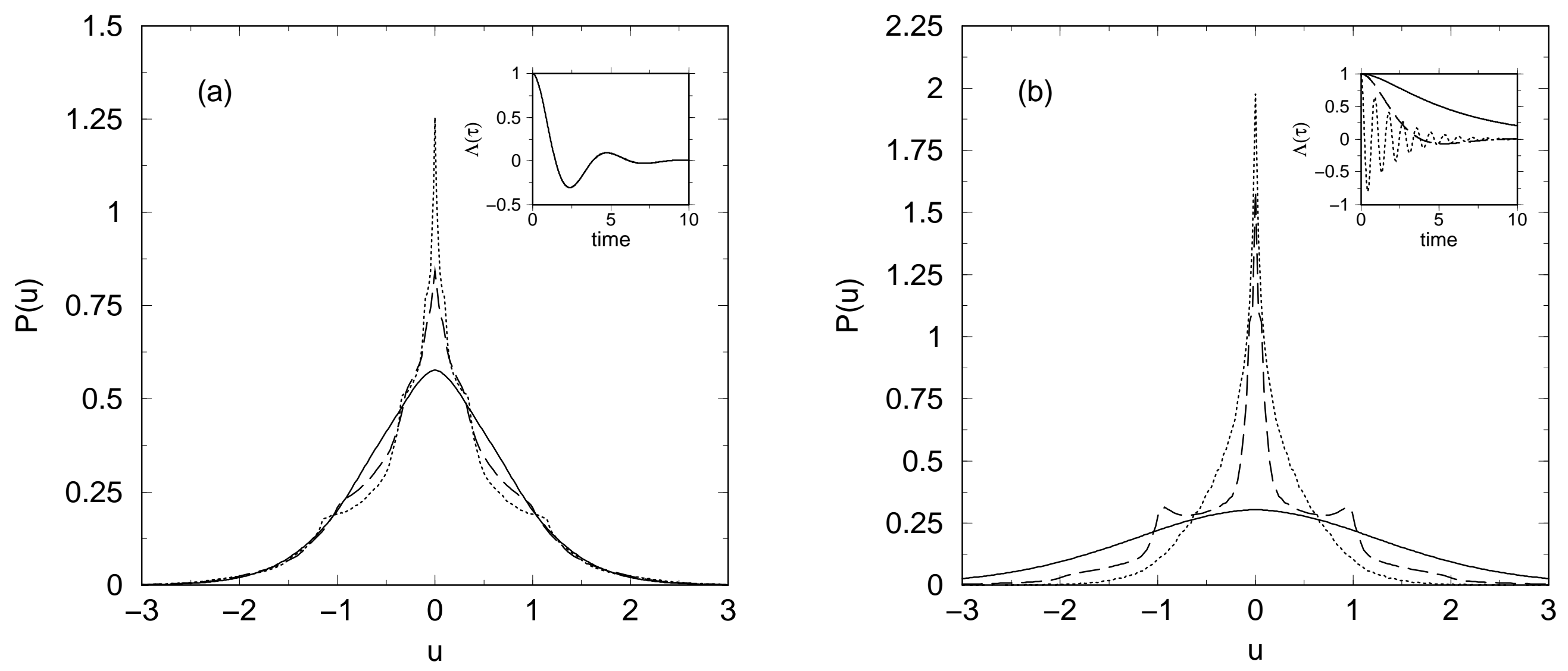